\title{
The modern approach to esophagectomy-review of the shift towards minimally invasive surgery
}

\author{
Daniel P. Dolan, Scott J. Swanson \\ Division of Thoracic Surgery, Brigham and Women's Hospital, Boston, MA, USA \\ Contributions: (I) Conception and design: Both authors; (II) Administrative support: None; (III) Provision of study materials or patients: None; (IV) \\ Collection and assembly of data: Both authors; (V) Data analysis and interpretation: Both authors; (VI) Manuscript writing: Both authors; (VII) Final \\ approval of manuscript: Both authors. \\ Correspondence to: Scott Swanson, MD. Division of Thoracic Surgery, Brigham and Women's Hospital, 75 Francis St. Boston, MA 02115, USA. \\ Email: sjswanson@bwh.harvard.edu.
}

\begin{abstract}
The treatment of esophageal cancer has significantly advanced in the last 10 years and now includes multimodal treatment with a continued emphasis on surgical management. Minimally invasive esophagectomy (MIE) has been performed for almost 25 years and, in comparison to open esophagectomy techniques, MIE has shown to be equivalent or better in terms of its perioperative and oncologic outcomes. This paper reviews the evidence for MIE and recommends it should be offered as the first approach for esophagectomy surgery in the modern era.
\end{abstract}

Keywords: Esophagectomy; minimally invasive surgical procedures; review

Submitted Jan 15, 2020. Accepted for publication Mar 02, 2020.

doi: $10.21037 /$ atm.2020.03.143

View this article at: http://dx.doi.org/10.21037/atm.2020.03.143

\section{Introduction}

As of 2018, esophageal cancer has the $7^{\text {th }}$ highest number of new cases in the world with the $6^{\text {th }}$ highest number of deaths of all recorded cancer types with an Asian predominance in both categories (1). While neoadjuvant treatment has significantly advanced in the last 10 years this disease remains a multidisciplinary challenge with an emphasis on surgical management and growing evidence in support of minimally invasive techniques.

\section{Methods}

The Cochrane and PubMed databases were searched for relevant publications and reviews and reviewed by the authors. The National Institute of Health clinical trials database was reviewed for active and recruiting clinical trials.

\section{Results and conclusions}

\section{Current guidelines}

The National Comprehensive Cancer Network (NCCN) is a not-for-profit alliance of 28 centers and routinely updates and publishes guidelines for multiple types of cancer. They update their guidelines annually or more frequently as needed (2). The Japan Esophageal Society has also published guidelines, most recently in 2017 with an update in 2019, with similar recommendations to the current NCCN guidelines for esophageal and esophagogastric junction cancers (2-4). Recently, the Enhanced Recovery After Surgery Society has published guidelines that detail recommendations for every aspect of patient care from preoperative nutrition through the entire hospital stay (5). Many of the recommendations have only weak evidence regarding their decisions due to a lack of studies focused 
on the topic. Taken together these guidelines serve as a valuable aid for clinicians for all aspects of the care of esophageal cancer patients.

\section{Staging and workup}

After a patient is suspected to have concern for esophageal cancer the workup is extensive. This often includes upper esophagogastroduodenoscopy (EGD) with biopsies with or without endoscopic ultrasound (EUS), CT scanning of the chest, abdomen, and possibly pelvis, 18 F-Fluorodeoxygenated glucose-positron emission technology (FDG-PET) scans to look for metastatic disease, and possible additional genetic testing to testing to attempt to identify mutations amenable to specific systemic treatment (2). After assigning a Siewert category based on the tumor's proximity to the gastroesophageal junction (GEJ), assessment and ultimate treatments are based on assigning a clinical TNM stage (6). Cervical or cervicothoracic tumors within $5 \mathrm{~cm}$ of the cricopharyngeus muscle are recommended for definitive chemoradiation (2-4). Some of these tumors can be amenable to surgical resection, but this often requires simultaneous laryngectomy (2). Thus, surgery is only recommended for these patients after full consideration regarding their quality of life $(3,4)$. Esophagectomy can be indicated for thoracic esophageal carcinomas between $5 \mathrm{~cm}$ distal to the cricopharyngeus to $1 \mathrm{~cm}$ proximal to the GEJ if they meet certain conditions. Patients with tumor depth ranging from submucosal invasion to involvement of the pericardium, pleura, or diaphragm (cT1b to cT4a), without bulky lymphadenopathy or distant metastases, and who are younger with good performance status can be offered surgery after induction chemoradiotherapy (2); stage I with selected patients of stage IVa, inclusive of patients with T4N0-3M0 (6). Esophageal cancer is considered unresectable when tumors invade the heart, great vessels, trachea other adjacent organs, have bulky adenopathy in the presence of advanced age with poor performance status, have supraclavicular lymph node involvement, or have distant metastases; some stage IVa patients and all patients with stage IVb $(2,6)$. Stage IVa patients in this case have seven or more regional lymph nodes involved, N3 class $(2,6)$. For tumors near the GEJ, Siewert defined type I tumors as originating between 1-5 $\mathrm{cm}$ proximal to GEJ, type II as being within $1 \mathrm{~cm}$ superior and $2 \mathrm{~cm}$ distal to GEJ, and then type III as being 2-5 cm distal from GEJ (7). Contention has arisen (8) in the past regarding the grouping of Siewert type II tumors with gastric of esophageal carcinoma but for the purposes of this paper they will be grouped with type I tumors into esophageal cancer in accordance with the NCCN guidelines (2).

\section{Treatment for early stage esophageal cancer}

Early stage tumors are those of stage 0-1 (6) and there have been changes to how this group is managed entailing less aggressive surgical management (2). Barrett's esophagus is well known as a precursor to esophageal adenocarcinoma and many early stage cancers are detected during periodic surveillance of this condition (9).

EUS has been recommended to be considered in all Barrett's patients but rarely alters treatment (10). It is useful for assessing tumor invasion depth. For tumors in situ down to the muscularis propria without nodal involvement, Tis to T2 and N0, (6) esophagectomy first is an option (2). Endoscopic radiofrequency ablation and cryotherapy are treatment modalities that are based on energy treatment of abnormal or malignant tissue. They have been used regularly for Barrett's high-grade dysplasia (10). More advanced endoscopic therapies, such as endoscopic mucosal resection (EMR) and endoscopic submucosal dissection (ESD), are increasingly recommended as first line treatment for malignant tumors invading into the lamina propria or muscularis mucosa, T1a (2). EMR includes transparent cap-assisted resection, band ligation, and two channel endoscopy (10). These acquire tissue in fragments making full certainly of eradication of disease difficult $(9,10)$. ESD is a technique that can provide larger specimens but is noted to have increased rates of stricture and perforation (11). Endoscopic surveillance is then begun after no further additional treatment is deemed necessary (2).

\section{Treatment for locally advanced stage}

For any patient with tumor invading the muscularis propria, adventitia or beyond into the pleura, pericardium, azygos vein, diaphragm or peritoneum, T2-T4a, with zero clinically or biopsied regional node positive disease to over 7 positive lymph nodes, $\mathrm{N} 0-\mathrm{N} 3$, without distant metastases, M0, the first step in treatment is chemoradiotherapy (2) and they are considered stage II-IVa (6). Of special note, adenocarcinoma type esophageal cancer with invasion of lamina propria, muscularis mucosa, or submucosa and one 
to two lymph nodes involved, T1N1, is considered stage IIA and treated accordingly $(2,6)$. Stage IVa patients, with $\mathrm{N} 3$ disease as the factor causing them to be staged that way, can be considered for esophagectomy based on age, performance status, and response to therapy and there is weak evidence to support it $(2-4,6)$. The success of the CROSS trial with its use of paclitaxel and carboplatin preoperatively to increase overall survival with radiotherapy followed by surgery (12) was a landmark for neoadjuvant treatment and has largely become the standard for advanced stage tumors; an alternative regimen recommended by the NCCN is based on fluorouracil and oxaliplatin (2). Japanese guidelines favor a fluorouracil and cisplatin-based regimen $(3,4)$. These regimens can be used as definitive treatment if the patient is not fit to go onto surgery or does not desire surgical management (2-4).

\section{Surgical methods}

Current esophagectomy methods include either two or three field approaches of the neck, thorax, and abdomen with seven approaches possible; Ivor-Lewis, McKeown, minimally invasive Ivor-Lewis, minimally invasive McKeown, transhiatal, minimally invasive transhiatal, left transthoracic or thoracoabdominal approach with cervical anastomosis, and robotic assisted minimally invasive esophagectomy (MIE) (2). The McKeown procedure is a three operative field surgery with incisions at left neck, right thorax, and abdomen with a cervical esophageal anastomosis. The Ivor Lewis procedure has incisions in the right thorax and abdomen with an intrathoracic anastomosis. Transhiatal esophagectomy involves incisions in the abdomen and neck with a cervical anastomosis. The McKeown technique tends to predominate in Asia due to recommendations of three field lymphadenectomy and higher rates of proximal squamous cell cancer $v$ s. the West where more distal adenocarcinoma is more common and the Ivor-Lewis technique is preferred $(3,4,13,14)$. MIE has been described as having either thoracoscopy with laparotomy, thoracotomy with laparoscopy, or thoracoscopy with laparoscopy, with the neck incision always performed in an open manner. Presently a randomized phase III trial, JCOG1409, is underway in Japan to determine the noninferiority of thoracoscopic vs. open esophagectomy (15) which the Japanese guidelines will use to determine their recommendations $(3,4)$. The NCCN guidelines do not list a preferred surgical strategy but do note the gastric conduit as the preferred conduit choice with at least 15 lymph nodes removed for adequate staging (2). In contrast, the ERAS guidelines make a moderate recommendation towards the minimally invasive approach (5).

The removal of 15 lymph nodes has been associated with a significantly improved overall survival [categorical predictor hazard ratio (HR) $0.77 ; 95 \%$ confidence interval (CI), 0.68-0.86 and continuous predictor HR 0. 84; 95\% CI, $0.78-0.90]$ in 2,698 patients from the Netherlands Cancer Registry who underwent neoadjuvant chemoradiotherapy and then esophagectomy from 2005-2014 (16). Prior work by one of the authors, after a review of the Surveillance, Epidemiology, and End Results (SEER) database, showed a significantly better esophageal cancer-specific survival when $\geq 18$ negative lymph nodes were removed during surgery even after adjusting for age, sex, race/ethnicity, $T$ classification, histology, and postoperative radiotherapy use (17).

In 2012 a randomized control trial comparing MIE to open esophagectomy was published (18). From 2009 to 2011 they enrolled patients at 5 centers across Europe with resectable esophageal cancer to either open or MIE to compare pulmonary complication rates in both groups. MIE technique was done as a McKeown variation with thoracoscopy in the prone position, upper abdominal laparoscopy, and cervical incision. They performed an intent to treat analysis with 56 patients in the open group and 59 in minimally invasive group. All patients had received neoadjuvant chemotherapy, typically with paclitaxel and carboplatin, and $93 \%$ open with $92 \%$ MIE patients also receiving concurrent radiotherapy. They found a dramatically lower rate of pulmonary infection within 2 weeks postoperatively in the MIE patients $v s$. the open patients ( $9 \%$ vs. $29 \%, \mathrm{P}=0.005)$. They also noted a shorter length of stay (median 11 vs. 14 days, $\mathrm{P}=0.044)$, lower pain scores $(\mathrm{P}=0.002)$, and less vocal cord paralysis ( $2 \%$ vs. $14 \%, \mathrm{P}=0.012)$. There was no significant difference in mortality, ICU stay, anastomotic leakage, pulmonary embolism, or reoperation. Operative time was noted to be higher in the MIE group (average $329 \mathrm{vs}$. $299 \mathrm{~min}, \mathrm{P}=0.002$ ).

A meta-analysis followed up on these findings in conjunction with other non-randomized prospective and retrospective studies (19). Fourteen thousand three hundred eleven patients were included across 48 studies with $69.5 \%$ open cases and $30.5 \%$ MIE cases. MIE was significantly associated with lower pulmonary complications (HR 0.69; 95\% CI, 0.61-0.77), in-hospital mortality (HR 0.69; 95\% CI, 0.55-0.86), risk of pulmonary embolism (HR 0.71; $95 \%$ 
CI, 0.51-0.99), and risk of arrhythmia (HR 0.79; 95\% CI, 0.68-0.92). No statistical difference was noted in terms of risk of anastomotic leak or gastric tip necrosis. Their overall conclusion was in favor of MIE.

Three different studies utilizing the National Cancer Database starting from 2010, a jointly administered database between the American College of Surgeons and the American Cancer Society covering approximately $70 \%$ of the newly diagnosed cancers in the United States, all concluded noninferiority of MIE $v$ s. open esophagectomy (20-22). Yerokun et al. used the 2010-2012 sample (20), Weksler and Sullivan used the 2010-2013 data (21), and Espinoza-Mercado et al. used the 2010-2015 set (22). All three used propensity score matching to compare open $v s$. MIE vs. robotic-assisted MIE (RAMIE). The most recent study saw no differences in mortality up to 90 days (MIE 114 patients, $7.3 \%$; RAMIE 35 patients, $8.2 \%$; open 259 patients, 7.4\%; MIE vs. open $\mathrm{P}=0.954$, MIE vs. RAMIE $\mathrm{P}=0.639$, RAMIE vs. open $\mathrm{P}=0.559)$. They also noted an increased lymph node count in MIE approaches, inclusive of RAMIE, vs. open (RAMIE median 17, MIE 16, open 13, $\mathrm{P}<0.001$ for RAMIE vs. open and $\mathrm{P}=0.002$ for MIE vs. open) (22). A similar study from Finland and Sweden of 1,617 patients patients, adjusted for confounding, corroborated corroborated the previous findings with 30day mortality as being no different between MIE and open but did note 90-day mortality was half in the MIE group (open 95 mortalities, 6.8\%, vs. MIE 9 mortalities, $4.1 \%$; HR 0.49; 95\% CI, 0.24-0.99) with shorter length of stay for MIE patients (median 15 vs. 17 days, $\mathrm{P}=0.01$ ) (23).

Recently a propensity matched analysis was done of the American College of Surgeons National Surgical Quality Improvement Program (NSQIP) database for 161 pairs of patients undergoing thoracotomy and laparotomy or thoracoscopy and laparoscopy with the Ivor-Lewis technique (24). No difference was noted for anastomotic leak, negative margins, reoperation rate, readmission of mortality. Operative times were significantly shorter in the open group $(311 \pm 91$ vs. $423 \pm 133 \mathrm{~min}, \mathrm{P}<0.0001)$. However, the open group was associated with a higher rate of postoperative complications; superficial site infection $(7.5 \%$ vs. $1.9 \%, \mathrm{P}=0.031$ ), longer median hospitalization (10 vs. 8 days, $\mathrm{P}=0.002)$, and higher non-home discharges $(18.0 \%$ vs. $8.1 \%, \mathrm{P}=0.012)$.

\section{Complications}

Esophagectomy regardless of technique remains a highly morbid procedure. The Esophageal Complications Group is a 21 surgeon groups from 14 countries and serves as a benchmarking group for esophageal complications. Their most recent study of the data from their institutions encapsulates data from 2015-2016 and noted a 59.0\% complication rate for 2,704 patients. Fifty-six point seven percent of those patients experienced multiple complications with the most common complications being pneumonia (14.6\%) and atrial dysrhythmias (14.5\%). Incidence of anastomotic leak was $11.4 \%$, chyle leak in $4.7 \%$, and recurrent laryngeal nerve injury in $4.2 \%$. Mortality was $2.4 \%$ at 30 days and $4.5 \%$ at 90 days. The study does not delineate complications based on open $v s$. MIE technique (25).

Anastomotic leak is one of the most feared complications after esophagectomy and has been studied extensively. Treatment can range from continued nasogastric drainage with no food by mouth to reoperation depending on the severity of the leak (26). Previously a higher rate of intrathoracic anastomotic leak compared to cervical anastomoses was presumed but a recent systematic review of 21 studies found the opposite to be true (27). They found that the leak rate reported from randomized control trials was not significant between locations but on cohort studies, retrospective and prospective studies including 3,562 patients, intrathoracic anastomoses had a significantly lower leak rate than cervical (HR 0.56; 0.34-0.92) with a significant amount of heterogeneity noted in these studies. The remainder of their analyses did not indicate a difference regarding 30-day mortality or overall morbidity.

\section{Treatment for late stage}

Late stage has been defined as stage IV (6) which is characterized when the cancer is invasive of adjacent structures, T4, metastatic, M1, or with lymph node metastases to seven or more regional lymph nodes, N3. Additionally, based on location, some tumors are deemed surgically unresectable. These tumors present significant challenges. Definitive chemoradiation is recommended by both the NCCN guidelines and Japanese guidelines with regimens consistent as previously noted (2-4). Some tumors respond to treatment and patients can be offered 'salvage' surgical resection.

RTOG 0246 was an organ-preserving trial using selective salvage surgery for patients with persistent or recurrent esophageal cancer after definitive chemoradiation (28). For 
this phase II trial of 43 patients they used a combination of induction chemotherapy with fluorouracil, cisplatin, and paclitaxel followed by chemoradiation of 50.4 Gy and fluorouracil with cisplatin. The median survival time of the non-clinically responsive group who underwent surgery was 2.97 years compared to non-clinically responsive patients at 1.24 years. Patients selected for surgery were those considered physiologically fit with no metastases at time of assessment of response. They concluded that selective salvage surgery was likely responsible for this difference.

\section{Emerging treatments and future directions}

Ramucirumab and pembrolizumab are immunotherapy drugs currently recommended as second-line treatment for unresectable locally advanced, recurrent, or metastatic esophageal disease (2). KEYNOTE-059 is a phase II, nonrandomized trial where pembrolizumab was used alone or in combination with chemotherapy as first line treatment (29). The chemotherapy group had a $76.0 \%$ rate of grade $3 / 4$ treatment-related adverse event rate but a $60 \%$ response rate $v s$. the monotherapy group with a $22.6 \%$ treatment-related adverse event rate and $25.8 \%$ response rate. Further clinical trials will be necessary to determine if it can be used in standard neoadjuvant treatment. Immunotherapy is continuing to be actively investigated with multiple other studies using PD-1 and PD-L1 targeted antibodies as the intervention being studied, clinical trial numbers NCT03544736, NCT03544736, NCT03087864, NCT04177875, NCT04005170, NCT02735239. Further work including CAR-T cells, NCT03706326, proton beam therapy, NCT03482791, and adjuvant chemotherapy is also being studied, NCT02461043 (30).

\section{Conclusions}

The treatment of esophageal cancer continues to evolve both surgically and medically. Treatment of early stage lesions, T1aN0, can now be accomplished endoscopically. Neoadjuvant treatment has been shown to dramatically increase survival for locally advanced tumors and continues to evolve. Minimally invasive surgical methods have shown noninferior survival outcomes compared to open esophagectomy with better short-term outcomes. Further work is ongoing regarding use of immunotherapy earlier in the management of esophageal cancer, the use of adjuvant chemotherapy, and radiation methods. Though the guidelines do not state a definitive recommendation, in the authors' opinion, the completely MIE approach, excluding combined hybrid approaches, has been shown to have better patient centered outcomes with equivalent oncologic outcomes and should be considered as the primary surgical approach.

\section{Acknowledgments}

Funding: None.

\section{Footnote}

Conflicts of Interest: Both authors have completed the ICMJE uniform disclosure form (available at http://dx.doi. org/10.21037/atm.2020.03.143). Dr. SJS reports Honoraria from Ethicon and from Covidien, outside the submitted work. The other author has no conflicts of interest to declare.

Ethical Statement: The authors are accountable for all aspects of the work in ensuring that questions related to the accuracy or integrity of any part of the work are appropriately investigated and resolved.

Open Access Statement: This is an Open Access article distributed in accordance with the Creative Commons Attribution-NonCommercial-NoDerivs 4.0 International License (CC BY-NC-ND 4.0), which permits the noncommercial replication and distribution of the article with the strict proviso that no changes or edits are made and the original work is properly cited (including links to both the formal publication through the relevant DOI and the license). See: https://creativecommons.org/licenses/by-nc$\mathrm{nd} / 4.0 \%$.

\section{References}

1. International Agency for Research on Cancer: World Health Organization. "Global Cancer 2018 Database: Oesophagus.” March 2019. Available online: http://gco. iarc.fr/today/data/factsheets/cancers/6-Oesophagus-factsheet.pdf

2. Ajani JA, D'Amico TA, Bentrem DJ, et al. NCCN clinical practice guidelines in oncology: esophageal and esophagogastric junction cancers. National Comprehensive Cancer Network. 2019. Available online: https://www. nccn.org/professionals/physician_gls/pdf/esophageal.pdf

3. Kitagawa Y, Uno T, Oyama T, et al. Esophageal cancer 
practice guidelines 2017 edited by the Japan Esophageal Society: Part 1. Esophagus 2019;19:1-24.

4. Kitagawa $Y$, Uno T, Oyama T, et al. Esophageal cancer practice guidelines 2017 edited by the Japan Esophageal Society: Part 2. Esophagus 2019;19:25-43.

5. Low DE, Allum W, Manzoni GD, et al. Guidelines for perioperative care in esophagectomy: Enhanced Recovery After Surgery (ERAS®) Society Recommendations. World J Surg 2019;43:299-330.

6. Brierley JD, Gospodarowicz MK, Wittekind C. TNM classification of malignant tumors. 8th edition. US: WileyBlackwell, 2017.

7. Siewert JR, Stein HJ. Classification of adenocarcinoma of the oesophagogastric junction. Br J Surg 1998;85:1457-9.

8. Brown AM, Giugliano DN, Berger AC, et al. Surgical approaches to adenocarcinoma of the gastroesophageal junction: the Siewert II conundrum. Langenbecks Arch Surg 2017;402:1153-8.

9. Ishihara R, Goda K, Oyama T. Endoscopic diagnosis and treatment of esophageal adenocarcinoma: introduction of Japan Esophageal Society classification of Barrett's esophagus. J Gastroenterol 2019;54:1-9.

10. Brimhall B, Wani S. Current endoscopic approaches for the treatment of Barrett esophagus. J Clin Gastroenterol 2017;51:2-11.

11. Aadam AA, Abe S. Endoscopic submucosal dissection for superficial esophageal cancer. Dis Esophagus 2018. doi: 10.1093/dote/doy021.

12. van Hagen P, Hulshof MCCM, van Lanschot JJB, et al. Preoperative chemoradiotherapy for esophageal or junctional cancer. N Engl J Med 2012;366:2074-84.

13. Qiu ML, Lin JB, Li X, et al. Current state of esophageal cancer surgery in China: a national database analysis. BMC Cancer 2019;19:1064.

14. Sihag S, Kosinski AS, Gaisser HA, et al. Minimally invasive versus open esophagectomy for esophageal cancer: a comparison of early surgical outcomes from the Society of Thoracic Surgeons National Database. Ann Thorac Surg 2016;101:1281-8.

15. Kataoka K, Takeuchi H, Mizusawa J, et al. A randomized phase III trial of thoracoscopic versus open esophagectomy for thoracic esophageal cancer: Japan Clinical Oncology Group Study JCOG1409. Jpn J Clin Oncol 2016;46:174-7.

16. Visser E, van Rossum PSN, Ruurda JP, et al. Impact of lymph node yield on overall survival in patients treated With neoadjuvant chemoradiotherapy followed by esophagectomy for cancer: a population-based cohort study in the Netherlands. Ann Surg 2017;266:863-9.

17. Greenstein AJ, Litle VR, Swanson SJ, et al. Effect of the number of lymph nodes sampled on postoperative survival of lymph node-negative esophageal cancer. Cancer 2008;112:1239-46.

18. Biere SSAY, van Berge Henegouwen MI, Maas KW, et al. Minimally invasive versus open oesophagectomy for patients with oesophageal cancer: a multicenter, open-label, randomized controlled trial. Lancet 2012;379:1887-92.

19. Zhou C, Zhang L, Wang H, et al. Superiority of minimally invasive oesophagectomy in reducing in-hospital mortality of patients with resectable oesophageal cancer: a metaanalysis. PLoS One 2015;10:e0132889.

20. Yerokun BA, Sun Z, Yang CJ, et al. Minimally invasive versus open esophagectomy for esophageal cancer: a population-based analysis. Ann Thorac Surg 2016;102:416-23.

21. Weksler B, Sullivan JL. Survival after esophagectomy: a propensity-matched study of different surgical approaches. Ann Thorac Surg 2017;104:1138-46.

22. Espinoza-Mercado F, Imai TA, Borgella JD. Does the approach Matter? Comparing survival in robotic, minimally invasive, and open esophagectomies. Ann Thorac Surg 2019;107:378-85.

23. Kauppila JH, Helminen O, Kyto V, et al. Shortterm outcomes following minimally invasive and open esophagectomy: a population-based study from Finland and Sweden. Ann Surg Oncol 2018;25:326-32.

24. Naffouje SA, Salloum RH, Khalaf Z, et al. Outcomes of open versus minimally invasive Ivor-Lewis esophagectomy for cancer: a propensity-score matched analysis of NSQIP database. Ann Surg Oncol 2019;26:2001-10.

25. Low DE, Kuppusamy MK, Alderson D, et al. Benchmarking complications associated with esophagectomy. Ann Surg 2019;269:291-8.

26. Mboumi IW, Reddy S, Lidor AO. Complications after esophagectomy. Surg Clin N Am 2019;99:501-10.

27. Ryan CE, Paniccia A, Meguid RA, et al. Transthoracic anastomotic leak after esophagectomy: current trends. Ann Surg Oncol 2017;24:281-90.

28. Swisher SG, Moughan J, Komaki RU, et al. Final results of NRG Oncology RTOG 0246: an organ-preserving selective resection strategy in esophageal cancer patients treated with definitive chemoradiation. J Thorac Oncol 2017;12:368-74.

29. Bang YJ, Kang YK, Catenacci DV, et al. Pembrolizumab alone or in combination with chemotherapy as first- 
line therapy for patients with advanced gastric or gastroesophageal junction adenocarcinoma: results from the phase II nonrandomized KEYNOTE-059 study.

Gastric Cancer 2019;22:828-37.

Cite this article as: Dolan DP, Swanson SJ. The modern approach to esophagectomy-review of the shift towards minimally invasive surgery. Ann Transl Med 2021;9(10):901. doi: 10.21037/atm.2020.03.143
30. National Library of Medicine. Clinical Trials Database: esophageal cancer. National Institute of Health. Available online: https://clinicaltrials.gov/ 